\title{
Localization Changes and Possible Roles of Small GTPase Ran in Mouse Oocyte Maturation
}

Q. Y. Sun*, Y. K. Cao,* and Heide Schatten**

* Institute of Zoology, The Chinese Academy of Sciences, Beijing, 100080, China

**Dept. of Vet. Pathobiol., University of Missouri-Columbia, MO 65211

The 24kDa small GTPase Ran (Ras-related Nuclear)[1], whose cDNA sequence codes for 216 amino acid residues, was originally identified as TC4 by screening a human teratocarcinoma cDNA library with degenerate oligonucleotides based on the conserved DTAGQE sequence of Ras family members [2]. In this study, we investigated the localization and possible roles of Ran during mouse oocyte meiotic maturation by using immunostaining and confocal laser scanning microscopy, antibody microinjection and microtubule disturbance.

The results showed that Ran was localized mainly to the nucleus (except for the nucleolus) in the oocyte. At pro-metaphase of meiosis I, Ran distributed throughout the cell, but predominantly concentrated around the condensed chromosomes. During the completion of meiosis I, it concentrated to the meiotic spindle microtubules except for the midbody region [Fig. 1]. Ran s concentration around the spindle disappeared when microtubule assembly was inhibited by colchicine, while it was concentrated around the chromosomes after microtubule stabilization with taxol treatment. Ran did not display any role in cytokinesis during division when pseudo-cleavage of GV-intact oocytes was induced. Ran antibody microinjection delayed germinal vesicle breakdown, decreased the first polar body extrusion, and distorted spindle organization and chromosome alignment [Fig.2].

Our results indicate that Ran has a cell cycle-dependent localization and may have regulatory roles in cell cycle progression and microtubule organization during mouse oocyte maturation.

\section{References}

Y. Takai et al., Physiol Rev 81 (2001) 153.

G. Drivas et al., Mol Cell Biol 10 (1990) 1793. 

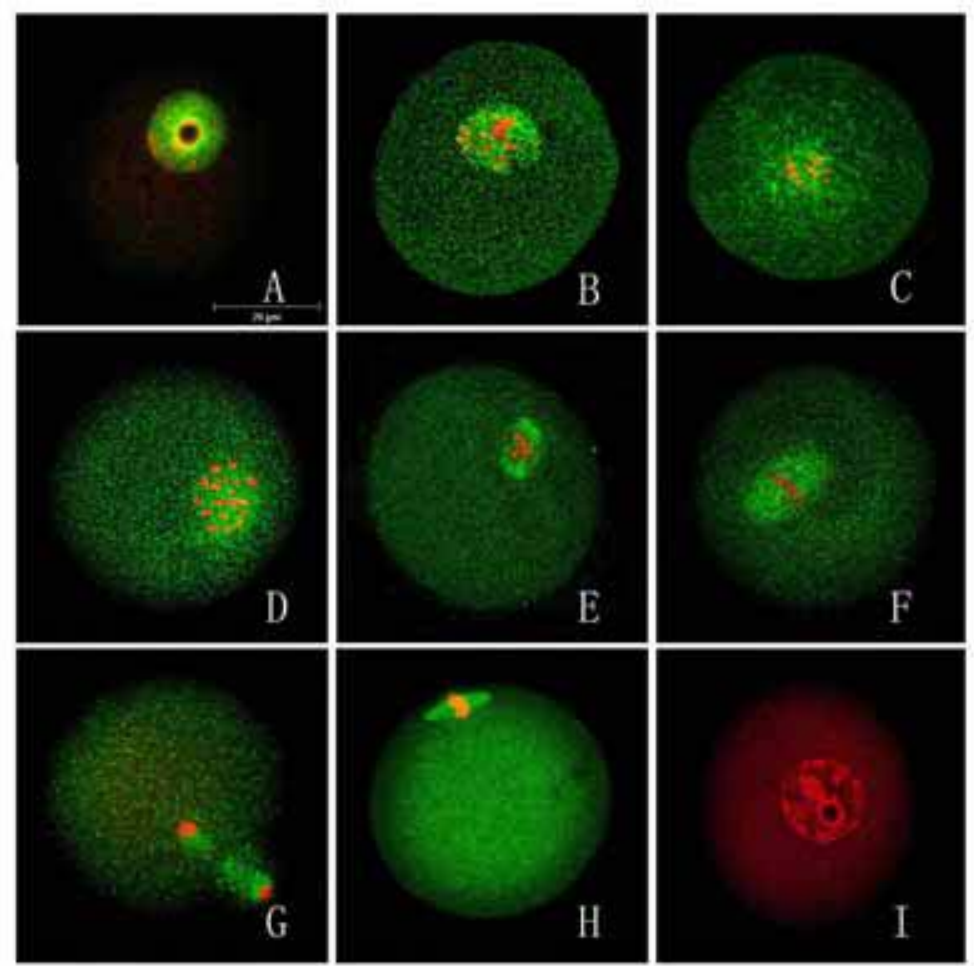

Fig. 1. Immunofluorescent localization of Ran during meiotic maturation. Green, Ran; red, chromatin; yellow, overlapping of green and red.

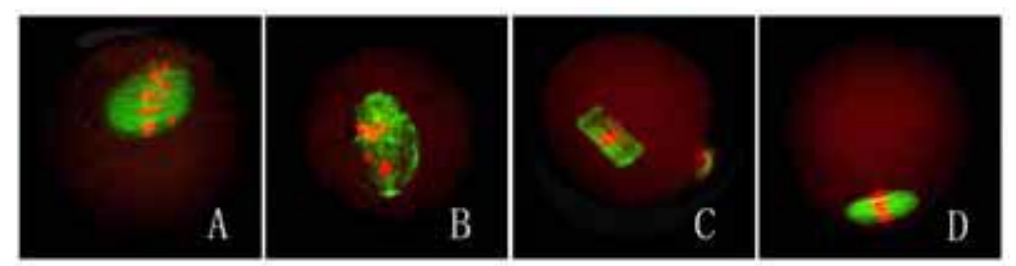

Fig.2. Effects of Ran antibody injection on spindle organization. (A) In most injected oocytes, the spindles were bigger and the chromosomes could not be organized to the equatorial plate. (B) A few oocytes did not form spindles. (C) The oocytes with the first polar body extruded showed abnormal spindles with broader poles. (D) Control. 